\title{
Enfermedades no transmisibles: factores de riesgo y acciones para su prevención y control en Cuba
}

\author{
Orlando Landrove-Rodríguez, ${ }^{1}$ Alain Morejón-Giraldoni, ${ }^{2}$ Silvia \\ Venero-Fernández, ${ }^{3}$ Ramón Suárez-Medina, ${ }^{3}$ Miguel Almaguer-López,, ${ }^{4}$ \\ Esther Pallarols-Mariño, ${ }^{5}$ Isora Ramos-Valle, ${ }^{1}$ Patricia Varona-Pérez, ${ }^{3}$ \\ Vivian Pérez-Jiménez ${ }^{6}$ y Pedro Ordúñez ${ }^{7}$
} $\begin{array}{ll}\text { Forma de citar } & \text { Landrove-Rodríguez O, Morejón-Giraldoni A, Venero-Fernández S, Suárez-Medina R, Almaguer-López M, } \\ \text { Pallarols-Mariño E, et al. Enfermedades no transmisibles: factores de riesgo y acciones para su prevención }\end{array}$ y control en Cuba. Rev Panam Salud Publica. 2018;42:e23. https:/ / doi.org/10.26633/RPSP.2018.23

RESUMEN Las enfermedades no transmisibles (ENT) representan uno de los mayores desafíos para el desarrollo en el siglo XXI, debido al devastador impacto social, económico y de la salud pública que provocan. El propósito de este artículo es describir la evolución y los factores de riesgo de las ENT en Cuba, principalmente en el período 1990-2015, reseñar las acciones emprendidas por el Ministerio de Salud Pública y destacar los desafíos más importantes para su prevención y control. La información contenida proviene de los datos colectados y publicados por la Dirección de Registros Médicos y Estadísticas de Salud, investigaciones sobre los factores de riesgo, otros estudios fundamentados y la documentación de acciones integrales. La mortalidad en Cuba está determinada por cuatro grandes problemas de salud: enfermedades cardiovasculares, tumores malignos, enfermedades crónicas de las vías respiratorias inferiores y diabetes mellitus, que en conjunto causan el $68,0 \%$ de los fallecimientos. La tendencia del cáncer es al ascenso y la enfermedad renal crónica emerge como un grave problema de salud. Cuba cuenta con una línea de base conocida sobre los factores de riesgo, de ellos la hipertensión y el consumo de tabaco son los principales relacionados con la mortalidad por ENT. En consonancia con la importancia de estas enfermedades se aprecian hitos e intervenciones de impacto positivo, así como brechas y desafíos en el marco del Plan de Acción Mundial de la Organización Mundial de la Salud para el enfrentamiento a las ENT.

Palabras clave

Enfermedad crónica; factores de riesgo; mortalidad prematura; determinantes sociales de la salud; Cuba.

Las enfermedades no transmisibles (ENT) representan un problema mundial, sobre todo para los países en vías de

\footnotetext{
Escuela Nacional de Salud Pública, La Habana, Cuba. Enviar la correspondencia a Orlando Landrove-Rodríguez, landrove@infomed.sld.cu, velandroor@gmail.com

2 Universidad de Ciencias Médicas de Cienfuegos, Cienfuegos, Cuba.

3 Instituto Nacional de Higiene, Epidemiología y Microbiología, La Habana, Cuba.
}

desarrollo, donde se han convertido en una importante carga para la salud pública en los últimos años.

\footnotetext{
4 Instituto de Nefrología, La Habana, Cuba

5 Ministerio de Salud Pública, La Habana, Cuba

Organización Panamericana de la Salud, La Habana, Cuba.

Organización Panamericana de la Salud, Enfermedades no Trasmisibles y Salud Mental, Washington DC, Estados Unidos de América.
}

Los avances económicos, sociales y sanitarios de Cuba a partir de la década de 1960 contribuyeron al desarrollo de un extenso y bien dotado sistema de salud pública que ha garantizado la cobertura universal y el acceso efectivo a los servicios de salud (1). Estos hechos derivaron en una situación epidemiológica y demográfica singular en un país en vías de desarrollo: una ostensible disminución de las enfermedades 
transmisibles y perinatales, con un predominio de las ENT y, como consecuencia, un gradual y acelerado envejecimiento de la población, con un nuevo perfil de enfermedades y mortalidad (2) que se asemeja al de los países desarrollados.

Para elaborar este artículo, se utilizaron los datos colectados por la Dirección de Registros Médicos y Estadísticas de Salud del Ministerio de Salud Pública (MINSAP) de Cuba, se evaluaron los resultados de la III Encuesta Nacional de Factores de Riesgo y Actividades Preventivas de las ENT (III ENFR), realizada en el 2010 (3) y de otros estudios documentados, y se analizaron las acciones integrales realizadas.

Para el estudio de las series y los análisis de mortalidad se tomaron en cuenta las listas con los códigos correspondientes para las enfermedades cardiovasculares (enfermedades del corazón, cerebrovasculares, y de las arterias, las arteriolas y los vasos capilares), los tumores malignos y la diabetes mellitus, según las diferentes revisiones de la Clasificación Internacional de Enfermedades (CIE-10 desde el 2001 hasta el 2015, la CIE-9 desde 1978 hasta el 2000 y la CIE-8 entre 1968 y 1977). En el caso de las enfermedades crónicas de las vías respiratorias inferiores, el período estudiado corresponde a 2001-2015, ya que a partir del 2001, cuando se comenzó a utilizar la CIE-10 en el país, esta nueva clasificación incorporó nuevos códigos de enfermedades —así como una mayor cantidad de estos - no considerados en las versiones anteriores. Por diversas razones, hasta la fecha, el MINSAP no ha podido reconstruir la serie de mortalidad ajustada por edad entre 1970 y el 2000, lo cual es una limitación en el presente estudio, aunque se pudo contabilizar los fallecidos totales por esta causa en el período 1990-2000.

El propósito de este artículo es describir la evolución de las ENT en Cuba y sus factores de riesgo, principalmente en el período 1990-2015, reseñar las acciones emprendidas por el MINSAP y destacar los desafíos más importantes para su prevención y control.

\section{ENVEJECIMIENTO Y CARGA DE LA ENFERMEDAD EN CUBA}

En el período 1994-1995, la esperanza de vida de los cubanos fue de 74,8 años, con un aumento según el cálculo más reciente (2011-2013) a 78,5 años (4).
En el año 1990, solo 11,9\% de los cubanos tenía 60 años de edad o más; en el 2015 ya este grupo representaba el $19,4 \%$ de la población. La tasa de mortalidad general ajustada por la edad en el 2015 fue de 4,7 defunciones por 1000 habitantes, valor inferior al 5,8 de 1990 (4).

En el 2015, los años de vida potencialmente perdidos en los grupos de edad comprendidos entre 1 y 74 años fueron 18,5 por cada 1000 habitantes por tumores malignos; 15,5 por enfermedades cardiovasculares; 1,8 por enfermedades crónicas de las vías respiratorias inferiores; y 1,4 por diabetes mellitus (4).

En 1990, a las enfermedades cardiovasculares, los tumores malignos, las enfermedades crónicas de las vías respiratorias inferiores y la diabetes mellitus se les atribuía el $64,1 \%$ del total de fallecidos; esta cifra aumentó a 68,0\% en el 2015 (figura 1). Ese último año, el $32,5 \%$ de los fallecidos en el país correspondió a muertes prematuras (entre 30 y 69 años) y, de estas, $68,7 \%$ se debió a las cuatro ENT mencionadas: los tumores malignos y las enfermedades cardiovasculares representaron el 32,9\% y el $29,5 \%$ de las muertes prematuras, respectivamente, mientras que las enfermedades crónicas de las vías respiratorias inferiores y la diabetes mellitus contribuyeron con $3,7 \%$ y $2,6 \%$, respectivamente (4).

\section{FIGURA 1. Mortalidad proporcional por causas seleccionadas. Cuba, 1990 y $2015^{\mathrm{a}}$}

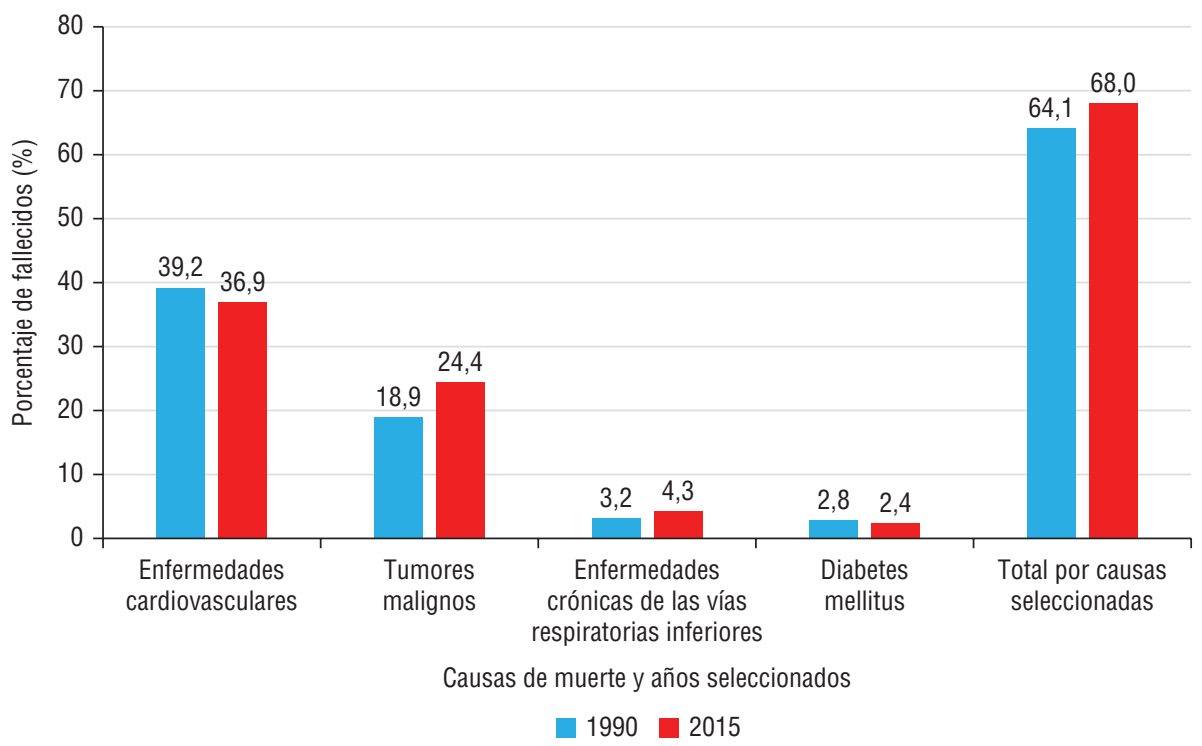

a Porcentaje que representan las causas seleccionadas en relación con el total de muertes registradas en Cuba en cada año escogido. En el caso de las enfermedades crónicas de las vías respiratorias inferiores, el período estudiado corresponde a 2001-2015.

Fuente: Elaborado por los autores a partir de la referencia 4 y datos de la Dirección Nacional de Registros Médicos y Estadísticas de Salud. 
reducción del índice de masa corporal de la población (6).

La mortalidad debida a las enfermedades del corazón ocurrió fundamentalmente por enfermedades cardíacas isquémicas $(69,1 \%)$, entre las que predominó el infarto agudo de miocardio (42,2\%) (4). La hipertensión arterial representó el mayor riesgo asociado a las enfermedades cerebrovasculares $(62,0 \%)$ y las enfermedades cardíacas isquémicas $(49,0 \%)$ (7).

Los tumores malignos se han mantenido como la segunda causa de muerte en el país desde finales de 1950 (8). La serie muestra una tendencia general al incremento de $0,42 \%$ anual, a pesar de que se observó un descenso anual de $1,16 \%$ entre 1970 y 1978 (figura 2). En el año 2015, esta causa representó el 24,4\% de las muertes del país, cifra mayor que la de 1990 (18,9\%) (figura 1). La mayoría de los tumores $(58,0 \%)$ se manifestó en localizaciones para las que ya existen programas preventivos: pulmón, tráquea y bronquios $(22,7 \%)$, próstata $(12,1 \%)$, colon $(9,7 \%)$, mama de la mujer $(6,4 \%)$, cavidad bucal $(3,2 \%)$, cuello del útero $(2,0 \%)$ y piel $(1,9 \%)$. Es elevado el riesgo de morir por tumores malignos localizados en la tráquea, los bronquios y el pulmón (tasa de 49,1 por 100000 habitantes) y el intestino - excepto el recto- (tasa de 20,6 por 100000 habitantes) (4), lo que evidencia brechas en los programas y una posible reserva para la reducción de la mortalidad por cáncer.

En el 2013, último año de notificación disponible, se registraron 44608 casos nuevos de cáncer. Las tasas ajustadas reflejan un predominio en los hombres (263,2 por 100000 habitantes frente a 223,1 por 100000 en las mujeres). Cada año se diagnostican más de 300 casos nuevos en menores de 20 años (4).

Las enfermedades crónicas de las vías respiratorias inferiores alcanzaron una tasa ajustada por la edad de 20,1 por 100000 habitantes, con una tendencia al ascenso desde el año 2001, reflejada en un ligero incremento anual de $0,79 \%$ (figura 3 ).

La mortalidad por asma ha disminuido sustancialmente en los últimos 25 años, asociado a mejoras en las intervenciones preventivas y terapéuticas; la tasa ajustada fue de 1,3 por 100000 habitantes en el $2015(4,9)$. El asma fue la tercera causa de consulta médica y egresos hospitalarios en el 2011 (9).

La diabetes mellitus presentó una reducción de la mortalidad en el período 1970-1980, con un decrecimiento anual de 0,63\%, aunque entre los años 1981 y 1992

FIGURA 2. Mortalidad por enfermedades cardiovasculares (ECV) y tumores malignos. Cuba, 1970-2015

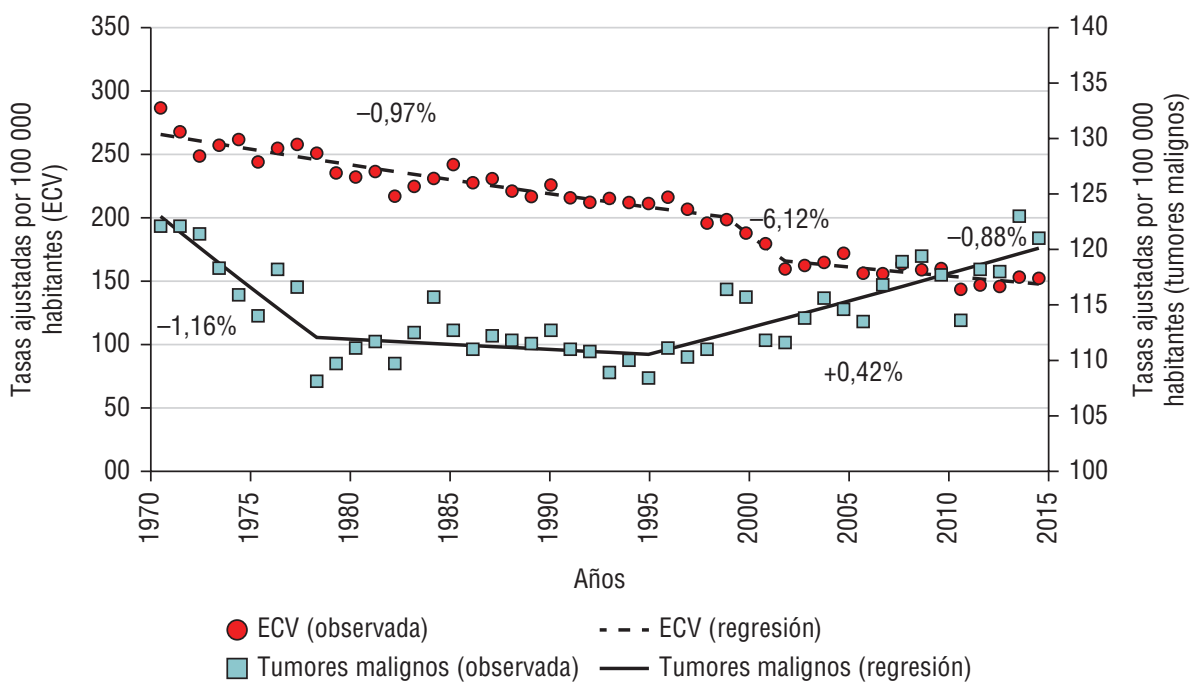

Fuente: Elaborado a partir de las referencias 4 y 5 , y datos de la Dirección Nacional de Registros Médicos y Estadísticas de Salud del Ministerio de Salud Pública de Cuba.

FIGURA 3. Mortalidad por enfermedades crónicas de las vías respiratorias inferiores (ECVRI) ${ }^{\mathrm{a}}$ y diabetes mellitus. Cuba, 1970-2015

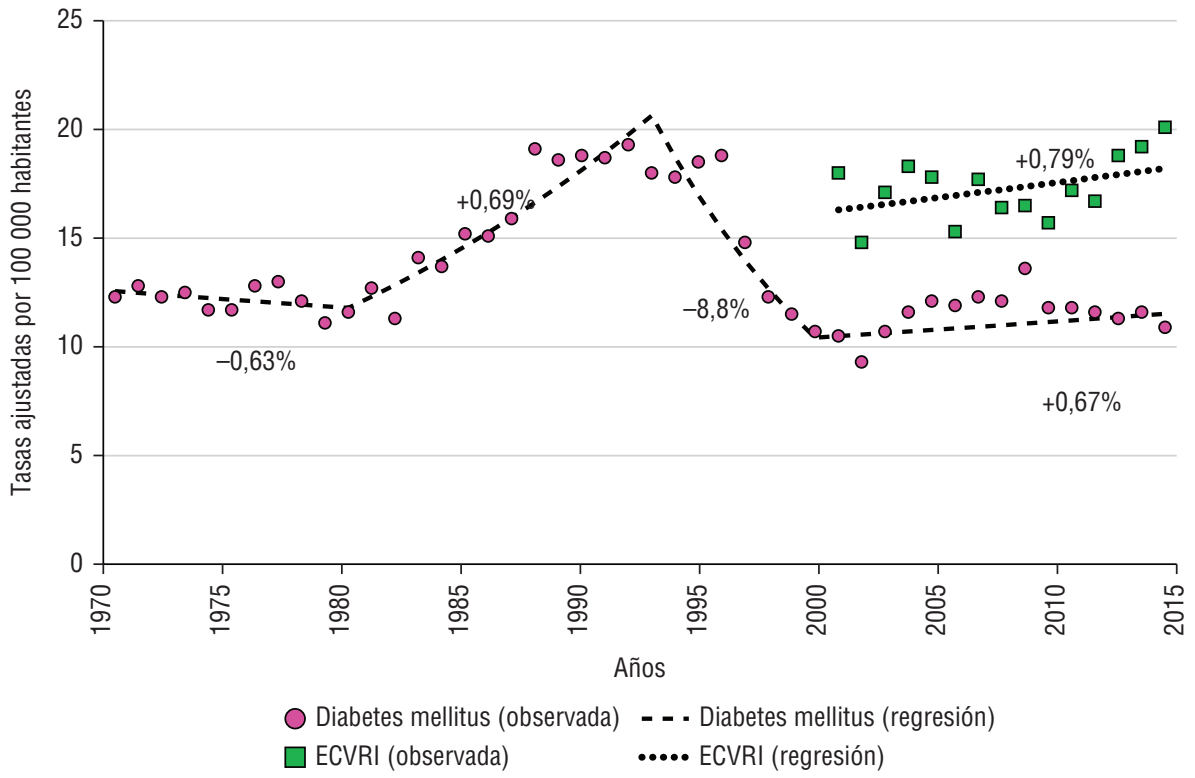

a No se dispone de datos para reconstruir la serie de mortalidad ajustada de las enfermedades crónicas de las vías respiratorias inferiores antes del 2001.

Nota: Según las diferentes revisiones de la Clasificación Internacional de Enfermedades (CIE-10 desde el 2001 hasta el 2015, la CIE-9 desde 1978 hasta el 2000 y la CIE-8 entre 1968 y 1977). Por diversas razones, hasta la fecha, el Ministerio de Salud Pública de Cuba (MINSAP) no ha podido reconstruir la serie de mortalidad ajustada por edad entre 1970 y el 2000.

Fuente: Elaborado a partir de las referencias 4 y 5, y datos de la Dirección Nacional de Registros Médicos y Estadísticas de Salud del MINSAP.

la tendencia fue al aumento (0,69\% anual). Entre los años 1993 y 2000 se observó un descenso importante $(8,8 \%)$, con un nuevo incremento anual de $0,67 \%$ entre el 2001 y el 2015 (figura 3). La tasa de mortalidad ajustada por la edad fue de 11,4 por 100000 habitantes en el 2015, más baja que en otros países de las Américas $(4,10)$.

\section{PRINCIPALES FACTORES DE RIESGO Y TENDENCIAS DE LAS ENFERMEDADES NO TRANSMISIBLES}

Cuba ha llevado a cabo tres encuestas poblacionales, representativas tanto a nivel provincial como nacional, para 
determinar los factores de riesgo en la población de 15 años de edad en adelante. La III ENFR compendia los resultados de las dos anteriores, realizadas en 1995 y el 2001 (3); estas encuestas han permitido contar con un sistema de base poblacional para la vigilancia de los factores de riesgo y conforman una sólida línea de base para la gestión de las ENT. En el municipio de Cienfuegos, sitio de demostración de la Iniciativa CARMEN (Conjunto de Acciones para la Reducción Multifactorial de Enfermedades No Transmisibles) en Cuba, se ha documentado la prevalencia de los factores de riesgo en los años 1991, 2001 y $2010(11,12)$.

Según los resultados de la III ENFR, se aprecia una tendencia al descenso del consumo de tabaco (36\% en 1995, 32\% en el 2001 y $24 \%$ en el 2010), aunque el ritmo de disminución fue solo de $0,8 \%$ anual (cuadro 1). La prevalencia de este factor de riesgo en las edades entre 13 y 15 años fue de $17,1 \%$, según la encuesta del año 2010 sobre consumo de tabaco en los jóvenes (13).

El consumo nocivo de alcohol también mostró una ligera tendencia al descenso. El mayor consumo correspondió a los hombres, sobre todo en las zonas urbanas y en los grupos comprendidos entre 25 y 54 años (3). La frecuencia de consumo perjudicial y la dependencia alcohóliencuestas realizadas (3) (cuadro 1).

Con respecto a la actividad física, se constata una tendencia al decrecimiento en los últimos 15 años, con un aumento ligero de la insuficiente actividad física población se clasificó como no activa (actividad física insuficiente), con mayor frecuencia en las mujeres, un resultado similar al de la encuesta del 2001. La insuficiente actividad física fue mayor durante el tiempo libre $(88,2 \%)$ (3). ca no varió sustancialmente entre las (3) (cuadro 1). En el 2010, el 39,6\% de la

En comparación con la encuesta realizada en el 2001, tanto el sobrepeso como la obesidad han aumentado (cuadro 1). La III ENFR mostró una prevalencia de hipertensión arterial de $31,9 \%$ en las áreas urbanas, inferior a la registrada en el 2001 (cuadro 1); en el 2010, 8,4\% de la población cubana padecía hipercolesterolemia (3).

La prevalencia de diabetes mellitus en Cuba fue de 10,1\% en la población de 15 años de edad o mayor (3), mientras que la enfermedad renal crónica emerge como un problema de salud creciente: según la III ENFR, 5,2\% de la población tiene una velocidad de filtración glomerular menor que $60 \mathrm{~mL} / \mathrm{min} / 1,73 \mathrm{~m}^{2}$ que indica una elevada sospecha de insuficiencia renal crónica (3). En un estudio poblacional realizado en el municipio Isla de la Juventud en el 2006 se encontraron valores similares (14).

En resumen, en la población cubana ha habido una tendencia al incremento de los factores de riesgo relacionados con las ENT. Este es un elemento que influye negativamente en la evolución de estas enfermedades.

\section{ACCIONES RELEVANTES DEL SISTEMA NACIONAL DE SALUD PARA LA PREVENCIÓN Y EL CONTROL DE LAS ENFERMEDADES NO TRANSMISIBLES}

Cuba tiene una reconocida historia en el campo de la salud, de la que forma parte su respuesta a las ENT. Aunque la mayoría de las acciones recogidas en este informe se ubican en el período 19902015, hay precedentes importantes como la creación de los institutos nacionales de salud en 1966, devenidos centros de investigación y docencia, cuya elevada calificación asistencial articularon en el

CUADRO 1. Prevalencia de factores de riesgo seleccionados. Cuba, 1995, 2001 y 2010a

\begin{tabular}{lccc}
\hline \multicolumn{1}{c}{ Factor de riesgo } & $1995^{\mathrm{b}}$ & $2001^{\mathrm{b}}$ & $2010^{\mathrm{b}}$ \\
\hline Consumo de tabaco & 36,0 & 32,0 & 24,0 \\
Consumo perjudicial y dependencia alcohólica & 8,0 & 7,7 & 7,8 \\
Sobrepeso (IMC de 25 a 29,9) & ND & 42,6 & 46,3 \\
Obesidad (IMC > 30) & ND & 11,8 & 15,4 \\
Actividad física insuficiente & 33,2 & 38,3 & 39,6 \\
Hipertensión arterial & 30,6 & 33,5 & 31,9 \\
\hline
\end{tabular}

a En porcentajes.

${ }^{b}$ Datos de poblaciones urbanas.

Nota: IMC: índice de masa corporal; ND: información no disponible.

Fuente: Elaborado por los autores a partir de los datos de la III Encuesta Nacional de Factores de Riesgo y Actividades Preventivas de Enfermedades no Transmisibles (3). sistema de salud las investigaciones relacionadas con las principales ENT (15). A partir de la década de 1970, se reforzó la ampliación continua de la cobertura de los servicios con políticas de inclusión social y con el desarrollo de la estrategia de atención primaria, consolidada en 1984 con el surgimiento del Programa del Médico y la Enfermera de la Familia (16); este programa favoreció la atención integral mediante el seguimiento activo y continuo de los pacientes y los familiares directamente en la comunidad. En ese año se creó el Departamento de Enfermedades Crónicas en el Instituto Nacional de Higiene, Epidemiología y Microbiología para el seguimiento integral de las ENT.

En 1992 se presentó el plan estratégico Objetivos, Propósitos y Directrices de la Salud Pública Cubana, previsto hasta el año 2000 (OPD 2000) (17), que por primera vez estableció las acciones y las metas integradas para enfrentar las ENT. En 1994 comenzó a funcionar el Departamento de Enfermedades no Transmisibles y el Centro de Promoción y Educación para la Salud, ambos ubicados en el MINSAP. Al mismo tiempo, se conformaron estructuras similares con personal especializado en las provincias y grupos de trabajo hasta el nivel de municipios. Esta organización incorporó nuevas tecnologías con un enfoque programático, fortaleció las acciones comunitarias e intersectoriales, promovió el enfoque multidisciplinario, consolidó la vigilancia de los factores de riesgo y de las ENT y contribuyó a la reorientación de la estrategia de atención primaria.

A partir del 2000 se implementó el Acuerdo 3790 del Comité Ejecutivo del Consejo de Ministros que aprobó el Programa de Salud y Calidad de Vida, basado en un modelo de participación intersectorial (18); en el 2006, y sobre la base de los resultados de los OPD 2000, se elaboraron las Proyecciones de la Salud Pública de Cuba hasta el año 2015 (19), que definieron los principales propósitos y metas para el período. Así, en el 2006 se constituyó la Sección de Cáncer en el MINSAP, cuyo objetivo es dirigir el Programa Integral de Control del Cáncer. En el 2009 se crearon dos secciones más: la sección de Salud Mental y Adicciones, con una red de centros de salud mental a nivel municipal, y la sección de Rehabilitación, que cuenta con 451 salas de rehabilitación física y con servicios de cesación tabáquica y consejería nutricional de base 
comunitaria en todos los policlínicos del país, un ejemplo formidable en términos de cobertura de los servicios de salud.

Desde el nivel central (MINSAP), estas unidades organizativas se integran en la gestión y la creación de capacidades, mediante la elaboración de programas, manuales, guías y otros recursos, con tecnología para la implementación de cada producto. Asimismo, toman en cuenta la actualización de la información a partir de las fuentes internacionales, el consenso de los grupos de asesores expertos en enfermedades prioritarias e incluye la acreditación académica para capacitar a los proveedores de salud. El Centro Nacional de Información de Ciencias Médicas y la Red de Salud de Cuba Infomed aportan las plataformas tecnológicas de las redes y los servicios. En esta red se encuentran los sitios de las instituciones de salud y los grupos especiales de trabajo por enfermedades priorizadas, y las redes de especialidades, entre otras, que permiten a los profesionales el acceso virtual a los productos desarrollados.

Varias redes facilitan la colaboración interinstitucional para la atención de las ENT: la Red de Cardiología y sus cardiocentros, la Red de Centros de Atención al Diabético, la Red de Unidades Nefrológicas y la Red de Unidades Oncológicas. Existen, además, 77 salas de terapia intensiva para adultos y 120 áreas intensivas municipales para garantizar los servicios de urgencia médica y el apoyo vital avanzado; la demanda principal de atención en estas unidades se deriva de los efectos de las ENT (20). Las instituciones biotecnológicas cubanas, integradas a la industria médico-farmacéutica, producen medios diagnósticos, reactivos, vacunas terapéuticas, equipos y más del 70\% de los medicamentos necesarios para el tratamiento de las ENT $(15,21)$. Estas instituciones contribuyen a las elevadas coberturas terapéuticas con medicamentos subvencionados por el Estado para pacientes con ENT y son un modelo de aplicación social de la investigación científica.

Numerosos ejemplos demuestran los resultados alcanzados por estas acciones. En los años 1998 y 2010, Cuba ejecutó campañas nacionales para mejorar el control de la hipertensión arterial. Se incrementó la detección de los hipertensos de 15 años o más registrados en los servicios de salud, que pasó de $8,8 \%$ en 1996 a $12,5 \%$ en 1998 , y se elevó a $25 \%$ en el año 2015 (4, 22). Estos resultados fueron corroborados en la III ENFR, pues el 73\% de los hipertensos conocía su condición, el $89 \%$ de los que conocían de su enfermedad recibían tratamiento y 55\% consiguió controlar su hipertensión (3); a nivel poblacional, el control de la hipertensión fue de 36\%. En las Américas solo en tres países el control de la hipertensión a nivel poblacional es superior a 35\% (Cuba, Estados Unidos de América y Canadá), que es el compromiso adoptado por los Estados Miembros de la Organización Panamericana de la Salud para el 2019 (3, 23).

Otro ejemplo de estos resultados notables es el alcanzado en el tratamiento de los diabéticos. El 75,5\% de los diabéticos recibió alguna medicación (tabletas o insulina) (3). Según un estudio realizado en la región central del país (24), las amputaciones del pie diabético — sufridas por más del $30 \%$ de los pacientes con úlceras del pie diabético, que en Cuba provocaban cerca de 1000 amputaciones de miembros inferiores cada año (25), para una tasa de 2 por cada 1000 diabéticos adultos registrados (3) - se han logrado reducir en $81,2 \%$ mediante la aplicación de Heberprot- $\mathrm{P}^{\circledR}$ (15), un producto biotecnológico cubano producido a partir del factor de crecimiento epidérmico humano recombinante, también desarrollado en Cuba.

Se sospecha que la prevalencia de insuficiencia renal en Cuba es elevada y el incremento anual de pacientes en terapia renal de remplazo dialítica es de $6 \%$ a $20 \%$. Más de 3000 pacientes reciben este servicio y se realizan más de 100 trasplantes renales al año (alrededor de 300 por millón de habitantes). Si se toma en cuenta la prevalencia de diabetes, hipertensión, obesidad, enfermedades renales primarias y el creciente envejecimiento poblacional (26), se concluye que aún se debe avanzar más en la detección precoz de esta enfermedad, por la consecuente pérdida en la oportunidad de tratamiento temprano.

Cuba dispone de un sólido sistema de vigilancia de factores de riesgo y ENT (27), con datos fiables integrados en el sistema de información estadística, además de los obtenidos mediante las tres encuestas nacionales de factores de riesgo (3). En el año 2011, quedó articulado en todas las provincias el sistema de vigilancia para los factores de riesgo, según la metodología de la Organización Mundial de la Salud (OMS) denominada STEPS $(28,29)$. Como componente esencial de la vigilancia, el Departamento de Enfermedades No Transmisibles del MINSAP conduce las evaluaciones de los servicios y las tecnologías que permiten valorar múltiples eventos y la calidad de los procesos (30). Asimismo, se ha confeccionado el marco nacional de vigilancia, que adecua al país las metas y los indicadores del Plan de Acción Mundial para la Prevención y el Control de las ENT 20132020, de la OMS (31).

\section{DESAFÍOS Y PRINCIPALES ACCIONES EN EL ENFRENTAMIENTO A LAS ENFERMEDADES NO TRANSMISIBLES}

Los retos en la atención de las ENT para el sistema de salud de Cuba son enormes y complejos. Uno de los más importantes es acelerar en todo el país la atención que las ENT reciben a nivel local, lo que requiere una mejor preparación del sistema de salud y de otros sectores para propiciar los cambios de actitudes de las personas, las familias y las comunidades, con la creación de entornos más saludables. Este escenario es favorecido por el contexto internacional y se articula con las estrategias y las propuestas de organismos internacionales, sociedades médicas, e instituciones científicas y académicas. En consonancia con las trasformaciones del sistema de salud y con las políticas sociales del Estado cubano, se promueve la salud en todas las políticas sectoriales y se ha propuesto el perfeccionamiento de las políticas y las normas para que promuevan estilos de vida más saludables a fin de disminuir los factores de riesgo que más afectan a la población cubana (32).

El consumo de tabaco es un componente muy importante en la carga de enfermedad del país (33) y las intervenciones han sido insuficientes para detener esta tendencia. Particularmente, la elevada prevalencia de consumo de tabaco entre los jóvenes requiere estrategias de salud diferenciadas dirigidas a este grupo de edad y se requieren acciones integradas que faciliten la implementación del Convenio Marco para el Control del Tabaco, de la OMS (34). Otra de las áreas de mayor prioridad es la organización del sistema de cuidados, que debe mejorar la integralidad de las acciones, enfocarse en el paciente con factores de riesgo y ENT, y que deben incluir la promoción 
del autocuidado y la autorresponsabilidad. El sistema de salud está trabajando para extender el modelo de cuidados crónicos (35) en armonía con las bases conceptuales del modelo de atención primaria de salud. Parte de este desafío es perfeccionar la atención a pacientes que padecen hipertensión arterial, diabetes mellitus, asma, enfermedad renal crónica y cáncer, enfermedades en las que es posible alcanzar mayores tasas de control y mejorar la prevención secundaria para contribuir a disminuir la mortalidad prematura y la mortalidad evitable por estas causas.

De acuerdo con las mejores evidencias, en Cuba se desarrolla el proyecto de reducción de riesgos de las enfermedades cardiovasculares, cuyo sitio demostrativo radica en la provincia de Matanzas y que se extiende a otras provincias; su propósito principal es mejorar los niveles de control de la hipertensión arterial. Este proyecto hace hincapié en la calidad de la atención, y se apoya en la definición de un algoritmo de tratamiento simplificado basado en la evidencia, medicamentos de alta calidad, un registro clínico que ayuda al monitoreo clínico y a la evaluación del desempeño, el trabajo en equipo más allá del médico, y la promoción de la participación del paciente y la comunidad. Este modelo está basado en un amplio consenso internacional y en los resultados obtenidos en Canadá y los Estados Unidos (36).

\section{LECCIONES APRENDIDAS}

Debido a la complejidad del problema, el enfrentamiento a las ENT ha sido difícil $\mathrm{y}$, con frecuencia, fragmentado y disperso. No obstante, en Cuba se han logrado avances sustanciales en la conducción de acciones integrales para atender las ENT

1. Cooper RS, Kennelly JK, Orduñez García P. Health in Cuba. Int J Epidemiol. 2006;35(4): $817-24$.

2. Landrove-Rodríguez O, Gámez-Bernal AI. Transición epidemiológica y las enfermedades crónicas no transmisibles en las Américas y en Cuba: el programa de intervención cubano. Reporte Tecnico Vigilancia. 2005;10(6). Disponible en: http://bvs.sld. $\mathrm{cu} /$ uats/rtv_files/2005/landrove.htm Acceso el 23 de enero de 2016.

3. Bonet-Gorbea M, Varona-Pérez P, ed. III Encuesta Nacional de Factores de Riesgo y Actividades Preventivas de Enfermedades

CUADRO 2. Lecciones aprendidas durante el proceso de enfrentamiento a las enfermedades no transmisibles (ENT)

1. La integración de las diferentes áreas, instituciones y disciplinas en el propósito de enfrentar las ENT es compleja, pero se ha logrado el consenso para la formación y la capacitación sistemática del capital humano, con la participación multidisciplinaria y la contribución de las instituciones académicas, lo cual ha permitido una mayor atención estandarizada de las ENT.

2. El desarrollo de las capacidades y los productos de la industria médico-farmacéutica y biotecnológica nacional, dirigidos a atender las necesidades crecientes de los pacientes con ENT, en consonancia con el envejecimiento poblacional, representa una fortaleza.

3. La conformación de un sistema de vigilancia desde el nivel nacional hasta el local aporta importantes insumos, que permiten la mejora continua de la gestión sobre la prevención y el control de las ENT y sus factores de riesgo; es necesario explotar estas posibilidades con mayor eficacia.

4. Afrontar de manera exitosa los desafíos que las ENT imponen al sistema de salud, exige estrategias igualmente eficaces y balanceadas para la prevención primaria y secundaria. En el contexto cubano significa, principalmente, fortalecer las políticas nacionales que favorecen la creación y el desarrollo de entornos saludables con una disminución significativa del consumo de tabaco y alcohol, y una mejoría del régimen de actividad física, así como una dieta saludable.

5. Se requiere un avance sustancial en el manejo clínico de las ENT, donde resultan críticos, entre otros factores, mejorar las tasas de control de la hipertensión, extender la cobertura de prevención secundaria de las enfermedades cardiovasculares y mejorar la calidad de las prestaciones para las personas con diabetes y enfermedades crónicas de las vías respiratorias. Igualmente, es necesaria una detección precoz eficaz de los tumores malignos prevenibles, con tratamientos oportunos y estandarizados.

6. Es necesario superar la limitada documentación de los procesos y las intervenciones exitosas en el campo de la prevención y el control de las ENT realizadas en el país.

Fuente: Elaborado por los autores.

mediante la creación de una estructura que va desde la instancia ministerial hasta el nivel local, favorecida por la implementación de la estrategia de atención primaria de salud y una cobertura realmente universal de los servicios. En el cuadro 2 se presentan las principales lecciones aprendidas durante el proceso de enfrentamiento a las ENT.

\section{CONCLUSIONES}

La mortalidad en Cuba está determinada por cuatro grandes problemas de salud: las enfermedades cardiovasculares, los tumores malignos, las enfermedades crónicas de las vías respiratorias inferiores y la diabetes mellitus, que en conjunto causan el $68,0 \%$ de los fallecimientos. La tendencia del cáncer es al ascenso y la enfermedad renal crónica emerge como un grave problema de salud. Cuba cuenta con una línea de base conocida sobre los factores de riesgo: la hipertensión y el consumo de tabaco son los principales factores de riesgo relacionados con la mortalidad por ENT. En consonancia con la importancia de estas enfermedades se aprecian hitos importantes e intervenciones de impacto positivo, así como brechas y desafíos en el marco del Plan de Acción Mundial para el Enfrentamiento a las ENT, de la OMS.

\section{Conflicto de intereses. Ninguno.}

Declaración. Las opiniones expresadas en este manuscrito son responsabilidad de los autores y no reflejan necesariamente los criterios ni la política de la Revista Panamericana de Salud Pública/Pan American Journal of Public Health o de la Organización Panamericana de la Salud.

\section{REFERENCIAS}

No Transmisibles. Cuba 2010-2011. La Habana: Editorial Ciencias Médicas; 2014. 4. Cuba Ministerio de Salud Pública. Anuario Estadístico de Salud 2016. La Habana: Dirección Nacional de Estadísticas; 2017. Disponible en: http:/ / www.sld.cu/sitios / dne Acceso el 28 de septiembre de 2017.

5. United States, National Cancer Institute, Surveillance Research Program. Joinpoint Regression Program, Version 4.1.1.4, February 2015; Statistical Methodology and Applications Branch. Washington, DC: NCI; 2015. Disponible en: http:/ / surveillance.cancer.gov/joinpoint/webhelp/
Getting_Started/citation.htm Acceso el 28 de marzo de 2017.

6. Franco M, Bilal U, Ordúñez P, Benet $M$, Morejón A, Caballero B, et al. Populationwide weight loss and regain in relation to diabetes burden and cardiovascular mortality in Cuba 1980-2010: repeated cross sectional surveys and ecological comparison of secular trends. Br Med J. 2013;346: f1515.

7. World Health Organization. Global health risks: mortality and burden of disease attributable to selected major risks. Geneva: WHO; 2009. Disponible en: http://www. 
who.int/healthinfo/global_burden_disease/GlobalHealthRisks_report_full.pdf Acceso el 28 de septiembre de 2017.

8. Camacho R, Fernández L, Martín A, Abascal ME, Díaz M. El programa nacional de control del cáncer en Cuba. Rev Cub Med Gen Integr. 1994;3(2):215-9.

9. Venero-Fernández SJ, González-Barcala FJ, Suárez-Medina R, Fabré-Ortíz D, FernándezNúñez HM. Epidemiology of asthma mortality in Cuba and its relation to climate, 1989 to 2003. MEDICC Review. 2008;10(3):24-9.

10. Ordúñez $P$, Prieto-Lara E, Pinheiro Gawryszewski V, Hennis AJM, Cooper RS. Premature mortality from cardiovascular disease in the Americas. Will the goal of a decline of " $25 \%$ by 2025 " be met? PLoS One. 2015;10(10):e0141685.

11. Ordúñez P, Barceló A, Bernal JL, Espinosa A, Silva LC, Cooper RS. Risk factors associated with uncontrolled hypertension: findings from the baseline CARMEN survey in Cienfuegos, Cuba. J Hypertens. 2008; 26(4):663-71.

12. Benet-Rodríguez $\mathrm{M}$, Morejón-Giraldoni $\mathrm{A}$, Espinosa-Brito A, Landrove-Rodríguez O, Peraza-Alejo D, Ordúñez-García P. Factores de riesgo para enfermedades crónicas en Cienfuegos, Cuba 2010. Resultados preliminares de CARMEN II. Medisur. 2010;8(2). Disponible en: http://scielo.sld.cu/scielo. php? script $=$ sci_arttext\&pid $=$ S1727897X2010000200010 Acceso el 28 de abril de 2017.

13. Pan American Health Organization. Youth and tobacco in the Americas. Results from the Global Youth Tobacco Survey 2000-2010. Washington, DC: PAHO; 2013. Disponible en: http:/ /www2.paho.org/hq/index.php? option $=$ com docman\&task $=$ doc view\&Itemid=270\&gid=24894\&lang=en Acceso el 2 de abril de 2017.

14. Almaguer-López M, Herrera-Valdés R, Chipi-Cabrera J, Toirac X, Castellanos O, Bacallao J. Design and methodology of the Isle of Youth community-based epidemiological study of CKD, cardio-cerebral vascular disease, hypertension, and diabetes mellitus (ISYS). MEDICC Review. 2007;9(1):23-30.

15. Rojo Pérez N, Valenti Pérez C, Martínez Trujillo N, Morales Suárez I, Martínez Torres E, Fleitas Estévez I, et al. Ciencia e innovación tecnológica en la salud en Cuba: resultados en problemas seleccionados. Rev Panam Salud Publica. 2018;42:e32. doi: 10.26633/RPSP.2018.32.

16. Cuba, Ministerio de Salud Pública. Programa del Médico y Enfermera de la Familia. La Habana: Editorial Ciencias Médicas; 2011. Disponible en: http://files.sld.cu/ sida / files / 2012 / 01 / programa-medico-y-enfermera-2011-vigente.pdf Acceso el 2 de abril de 2017.

17. Cuba, Ministerio de Salud Pública. Objetivos, propósitos y directrices de la salud pública cubana 1992-2000. La Habana: Editorial Ciencias Médicas; 1992.

18. García-Pérez RM, Pérez-González R, Landrove-Rodríguez O. Calidad de vida e indicadores de salud. Cuba, 2001-2002. Rev Cubana Hig Epidemiol. 2004;42(3). Disponible en: http://scielo.sld.cu/scielo. php? script $=$ sci_arttext $\&$ pid $=S 1561$ 30032004000300002 Acceso el 23 de febrero de 2017.

19. Cuba, Ministerio de Salud Pública. Proyecciones de la salud pública en Cuba para el 2015. La Habana: Editorial Ciencias Médicas; 2006.

20. Herrera JL, González R, Sosa A. Áreas intensivas municipales: estrategia vital para los servicios de urgencia en la atención primaria. Rev Cubana Med Int Emergen. 2008;7(2):1-38. Disponible en: http://bvs.sld.cu/revistas/mie/vol7_2 08/mie07208.pdf Acceso el 23 de abril de 2017.

21. Lage A. Connecting immunology research to public health: Cuban biotechnology. Immunol. 2008;9:109-112. Disponible en: http:/ / www.nature.com/articles/ni0208109 Acceso el 23 de abril de 2017.

22. Alfonso-Jorge $\mathrm{P}$, Landrove-Rodríguez $\mathrm{O}$, Pérez-Caballero D, Cordiés-Jackson L, Vázquez-Vigoa A, Dueñas-Herrera A. Hipertensión arterial en Cuba. Panorámica general de los últimos 25 años. Nefrol. 2000; 20(6):439.

23. Joffres M, Falaschetti E, Gillespie C, Robitaille C, Loustalot F, Poulter N, et al. Hypertension prevalence, awareness, treatment and control in national surveys from England, the USA and Canada, and correlation with stroke and ischaemic heart disease mortality: a cross-sectional study. BM] Open. 2013 Disponible en: http://bmjopen.bmj.com/content/bmjopen/3/8/ e003423.full.pdf Acceso el 30 de diciembre de 2015.

24. Fernández-Montequín JI, Betancourt BY, Leyva-González G, Mola EL, GalánNaranjo K, Ramírez-Navas M, et al. Intralesional administration of epidermal growth factor-based formulation (Heberprot-P) in chronic diabetic foot ulcer: treatment up to complete wound closure. Int Wound J. 2009;6(1):67-72.

25. González-Benavides C, Pérez-Mederos LM, Peraltas-Pérez G. Resultados de la utilización del Heberprot-P. Acta Med Cent. 2014;8(4). Disponible en: http://www.revactamedicacentro.sld.cu/index.php/ amc/article/view/192/pdf Acceso el 28 de abril de 2017.

26. Pérez-Oliva Díaz J, Portal-Miranda JA. Enfermedad renal crónica: estrategia nacional para enfrentar este importante problema de salud. Rev Hab Cienc Med. 2010;9(2). Disponible en: http://scielo.sld. $\mathrm{cu} /$ scielo.php? script $=$ sci_arttext\&pid $=$ S1729-519X2010000200001\&lng=es Acceso el 28 de abril de 2017.

27. Batista-Moliner R, Landrove-Rodríguez $\mathrm{O}$, Bonet-Gorbea $\mathrm{M}$, Feal-Cañizares $\mathrm{P}$, Ramírez-Rodríguez M. Sistema de vigilancia de enfermedades no transmisibles en Cuba. Rev Cubana Hig Epidemiol. 2000; 38(2):77-92.
28. World Health Organization. WHO STEPS surveillance manual: the WHO STEPwise approach to chronic disease risk factor surveillance / non communicable diseases and mental health. Geneva: WHO; 2007. Disponible en: http://apps.who.int/iris/ handle/10665/43376 Acceso el 28 de marzo de 2017.

29. Varona-Pérez P, Bonet-Gorbea M, Chang-De la Rosa M, Suárez-Medina R. Implementation of chronic disease risk factor surveillance in 12 Cuban municipalities. MEDICC Rev. 2014;16(1).

30. Rodríguez-Gavaldá D, Ramírez-Rodríguez $\mathrm{M}$, Landrove-Rodríguez O, SerranoVerdura C, Santín-Peña M, Valdivia-Onega C. EPICRONI: programa automatizado para el monitoreo y evaluación de la calidad de la atención médica de las enfermedades no transmisibles. Rev Cubana Hig Epidemiol. 2001;39(1):26-31.

31. World Health Organization. Global action plan for the prevention and control of noncommunicable diseases 2013-2020. Geneva: WHO; 2013. Disponible en: http://apps. who.int/iris/bitstream/10665/94384/ 1/9789241506236_eng.pdf Acceso el 28 de marzo de 2017

32. Partido Comunista de Cuba. Actualización de los Lineamientos de la Política Económica y Social del Partido y la Revolución para el periodo 2016-2021. La Habana: Editora Política; 2016. Disponible en: http://www.granma.cu/file/pdf/gaceta/ 01Folleto.Lineamientos-4.pdf Acceso el 22 de septiembre de 2017.

33. Varona-Pérez P, Herrera-D, García-Pérez R, Bonet-Gorbea M, Romero-Pérez T, VeneroFernández S. Smoking-attributable mortality in Cuba. MEDICC Rev. 2009;11(3): 43-7.

34. World Health Organization. Updated status of the WHO Framework Convention on Tobacco Control. Geneva: WHO; 2005. Disponible en: http://www.who.int/tobacco/framework/countrylist/en/index.html Acceso el 28 de septiembre de 2017.

35. Organización Panamericana de la Salud. Cuidados innovadores para las condiciones crónicas: organización y prestación de atención de alta calidad a las enfermedades crónicas no transmisibles en las Américas. Washington, DC: OPS; 2013.

36. Patel P, Orduñez P, Di Pette D, Escobar MC, Hasell T, Wyss F, et al. Improved blood pressure control to reduce cardiovascular disease morbidity and mortality: The Standardized Hypertension Treatment and Prevention Project. J Clin Hypertens (Greenwich). 2016;18(12):1284-94.

Manuscrito recibido el 30 de abril de 2017. Aceptado para publicación, tras revisión, el 17 de enero de 2018 
ABSTRACT Non-communicable diseases (NCDs) represent one of the greatest challenges for development of the 21st century due to their devastating social, economic and public health impact. The objective of this article are to describe the evolution and risk factors

Non-communicable diseases: risk factors and actions for their prevention and control in Cuba

Keywords for NCDs in Cuba, mainly in the period 1990-2015, to outline actions undertaken by the Ministry of Public Health of Cuba, and to highlight the most important challenges with a focus on their prevention and control. The information is based on data collected and published by the Directorate of Medical Records and Health Statistics, research on risk factors, other studies and documentation of comprehensive actions. Mortality in Cuba is determined by four major health problems: cardiovascular diseases, malignant tumors, chronic diseases of the lower respiratory tract and diabetes mellitus, which together cause $68.0 \%$ of deaths. Cancer presents a growing trend, and chronic kidney disease emerges as a serious health problem. Cuba has a known baseline on risk factors, and hypertension and tobacco consumption are the main factors related to NCDs mortality. In line with the importance of these diseases, there are milestones and interventions with a positive impact, as well as gaps and challenges within the framework of the World Health Organization's Global Action Plan for the Prevention and Control of NCDs.
RESUMO

Doenças não transmissíveis: fatores de risco e ações para sua prevenção e controle em Cuba

Palavras-chave
As doenças não transmissíveis (DNT) representam um dos maiores desafios do século 21 para o desenvolvimento devido ao desvastador impacto social, econômico e de saúde pública que elas causam. O objetivo deste artigo é descrever a evolução e os fatores de risco das doenças não transmissíveis em Cuba, principalmente no período de 1990-2015, para delinear as ações realizadas pelo Ministério da Saúde Pública de Cuba e destacar os desafios mais importantes para sua prevenção e controle. A informação vem dos dados coletados e publicados pela Diretoria de Registros Médicos e Estatísticas de Saúde, pesquisa sobre fatores de risco, outros estudos fundamentados e documentação de ações abrangentes. A mortalidade em Cuba é determinada por quatro principais problemas de saúde: doenças cardiovasculares, tumores malignos, doenças crônicas do trato respiratório inferior e diabetes mellitus, que em conjunto causam $68,0 \%$ das mortes. O câncer tem uma tendência crescente, e a doença renal crônica surge como um grave problema de saúde. Cuba tem uma linha de base conhecida sobre os fatores de risco, dos quais a hipertensão e o consumo de tabaco são os principais relacionados à mortalidade por DNT. Em consonância com a importância dessas doenças, há marcos e intervenções com impacto positivo, bem como lacunas e desafios no âmbito do Plano de Ação Mundial da Organização Mundial da Saúde para enfrentar as doenças não transmissíveis.

Doença crônica; fatores de risco; mortalidade prematura; determinantes sociais da saúde; Cuba. 